\title{
Frontocerebellar connectivity: climbing through the inferior olive
}

\author{
Eduardo Dias-Ferreira, ${ }^{1,2,}$, Nuno Sousa2 and Rui M. Costa ${ }^{1 *}$ \\ Champalimaud Neuroscience Programme at Instituto Gulbenkian de Ciência, Oeiras, Portugal \\ 2 Life and Health Sciences Research Institute, School of Health Sciences, University of Minho, Braga, Portugal \\ ${ }^{3}$ PhD Programme in Experimental Biology and Biomedicine, Center for Neuroscience and Cell Biology, University of Coimbra, Coimbra, Portugal \\ ${ }^{*}$ Correspondence: costarui@igc.gulbenkian.pt
}

\section{A commentary on}

Electrophysiological mapping of novel prefrontal - cerebellar pathways

by Thomas C. Watson, Matthew W. Jones and Richard Apps. Front. Integr. Neurosci. (2009), 3:18.

Is there a role of cerebellum beyond motor control? Neuroanatomical studies in nonhuman primates have shown connections between the cerebellum and non-motor cortical areas of the frontal lobe (Strick et al., 2009). These studies have not only supported the theoretical framework of how the cerebellum is involved in motor control but also forward models of frontocerebellar loop processing during cognitive processes (Ramnani, 2006). Interestingly, the larger prefrontal cortex of humans compared to non-human primates comes with enlarged cerebellar hemispheres, and the largest contribution to the cortical-cerebellar loops in the human brain does not seem to come from cortical motor areas, but from the prefrontal cortex (Ramnani, 2006). Sparse data mainly from clinical studies (Schmahmann et al., 2007) and fMRI in humans (Kim et al., 1994; Allen et al., 1997) have also contributed to the view that the cerebellum might be enrolled in higher cognitive functions. Still, the interpretation of the existing data is not consensual, and many of the studies involve oculomotor control (Glickstein, 2007) making it difficult to disentangle between motor and cognitive functions. Therefore, more integrative studies seem necessary to map the connectivity between the cerebellum and other brain areas, and to probe the causal relationships between circuit and behavior.

In a new study, Watson et al. (2009) put forward the existence of two distinct frontocerebellar pathways: one from the prelimbic (PrL) sub-region of medial prefrontal cortex (mPFC), and other from the premotor cortex (M2), projecting through the inferior olive before terminating in common climbing fibers in the contralateral vermis of lobule VII. Extensive mapping by recording evoked field potentials on the rat cerebellar surface during PrL stimulation revealed larger amplitude responses in the contralateral side, which decreased from medial to lateral positions, and showed greater responses in vermal lobule VII. Although this observation is supported by neuroanatomical data in the monkey (Kelly and Strick, 2003), other physiological studies would predict enhanced activity towards the lateral cerebellum (Sasaki et al., 1977). Even more interesting was the observation of features typical of climbing fiber field potentials, further confirmed by an increased probability of complex spike activity in single unit Purkinje cells of vermal lobule VII following PrL stimulation. These results support the existence of a cortico-olivary pathway as an alternative to the well documented cortico-pontine projections (Ramnani, 2006), and put forward the PrL sub-region of the mPFC as an interesting player in the corticocerebellar loop.

But could the PrL project to the cerebellum via other frontal cortex regions, for example M2? Watson et al. (2009) showed that M2 stimulation evoked similar topography of responses on the cerebellum as PrL stimulation. The largest field potentials evoked by M2 stimulation were also located in vermal lobe VII, and seemed to present the same features of climbing fiber field potentials (although the presence of complex spikes was not confirmed by singleunit recordings). Also, by looking into the mean latency of the responses, they showed that M2 stimulation evoked shorter latency responses than PrL stimulation. However, the longer latency responses evoked by PrL stimulation were not significantly affected by $\mathrm{M} 2$ reversible inactivation, suggesting that the observed climbing fiber responses arose from two frontocerebellar pathways with independent origins. Finally, the summation of responses resulting from suprathreshold simultaneous stimulation of PrL and M2 revealed that both frontocerebellar pathways share the same climbing fibers, and therefore should converge either before or at the inferior olive.

This study suggests two novel fronto-olivocerebellar pathways: a slower route leaving from the PrL cortex, probably going through the periaqueductal gray, and a $5 \mathrm{~ms}$ faster route from the M2 cortex, possibly taking a more direct pathway through the superior colliculus (as discussed by the authors). This is not just one more report describing descending projections from non-exclusively-motor areas in frontal cortex to the cerebellum (Ramnani, 2006; Strick et al., 2009), as it proposes a pathway through climbing fibers shown to induce LTD in the simultaneously activated parallel fiber-Purkinjecell synapses, and presumably to convey error signals to the circuit during action initiation and execution (Welsh et al., 1995; Ito, 2008). Taking these forward models into consideration, and the major players uncovered by this study, it is tempting to speculate about behavioral implications. First, these areas of the frontal cortex were shown to be involved in eyeblink conditioning (KronforstCollins and Disterhoft, 1998; Christian and Thompson, 2003), action initiation and termination (Fujii and Graybiel, 2003), and conflict-monitoring between more automatic and voluntary behavioral strategies (Isoda and Hikosaka, 2007). Second, some of these behaviors and other components, such as attention, engage directly or indirectly oculomotor control, which is one of the major functions attributed to the vermal lobule VII (Glickstein, 2007). Third, the output cerebellar nuclei to these frontal areas have been implicated in higher cognitive functions such 
as stimulus-outcome encoding (McCormick and Thompson, 1984), and automatization of recurrent actions (Lu et al., 1998). Considering these and other studies, the described frontocerebellar connections could be involved in the switch from voluntary to completely automatized behaviors and in skill learning, by providing automatic motor surveillance during the performance of actions or sequences of actions in a more precise and accurate manner. To test these and other hypothesis, further mapping studies are needed, in order to allow the efficient use of integrative approaches. Combining the analysis of behavior microstructure during specific tasks, online manipulation and recording of specific pathways, and looking into the functional connectivity between regions, will tell us more about the behavioral implications of the frontocerebellar interplay.

\section{REFERENCES}

Allen, G., Buxton, R. B., Wong, E. C., and Courchesne, E. (1997). Attentional activation of the cerebellum independent of motor involvement. Science 275, 1940-1943.
Christian, K. M., and Thompson, R. F. (2003). Neural substrates of eyeblink conditioning: acquisition and retention. Learn. Mem. 10, 427-455.

Fujii, N., and Graybiel, A. M. (2003). Representation of action sequence boundaries by macaque prefrontal cortical neurons. Science 301, 1246-1249.

Glickstein, M. (2007). What does the cerebellum really do? Curr. Biol. 17, R824-R827.

Isoda, M., and Hikosaka, O. (2007). Switching from automatic to controlled action by monkey medial frontal cortex. Nat. Neurosci. 10, 240-248.

Ito, M. (2008). Control of mental activities by internal models in the cerebellum. Nat. Rev. Neurosci. 9, 304-313.

Kelly, R. M., and Strick, P. L. (2003). Cerebellar loops with motor cortex and prefrontal cortex of a nonhuman primate. J. Neurosci. 23, 8432-8444.

Kim, S. G., Ugurbil, K., and Strick, P. L. (1994). Activation of a cerebellar output nucleus during cognitive processing. Science 265, 949-951.

Kronforst-Collins, M. A., and Disterhoft, J. F. (1998). Lesions of the caudal area of rabbit medial prefrontal cortex impair trace eyeblink conditioning. Neurobiol. Learn. Mem. 69, 147-162.

Lu, X., Hikosaka, O., and Miyachi, S. (1998). Role of monkey cerebellar nuclei in skill for sequential movement. J. Neurophysiol. 79, 2245-2254.

McCormick, D. A., and Thompson, R. F. (1984) Cerebellum: essential involvement in the classically conditioned eyelid response. Science 223, 296-299.

Ramnani, N. (2006). The primate cortico-cerebellar system: anatomy and function. Nat. Rev. Neurosci. 7 , 511-522.
Sasaki, K., Oka, H., Kawaguchi, S., Jinnai, K., and Yasuda, T. (1977). Mossy fibre and climbing fibre responses produced in the cerebeller cortex by stimulation of the cerebral cortex in monkeys. Exp. Brain Res. 29, 419-428.

Schmahmann, J. D., Weilburg, J. B., and Sherman, J. C. (2007). The neuropsychiatry of the cerebellum insights from the clinic. Cerebellum 6, 254-267.

Strick, P. L., Dum, R. P., and Fiez, J.A. (2009). Cerebellum and nonmotor function. Annu. Rev. Neurosci. 32 413-434.

Watson, T. C., Jones, M. W., and Apps, R. (2009). Electrophysiological mapping of novel prefrontal cerebellar pathways. Front. Integr. Neurosci. 3, 18.

Welsh, J. P., Lang, E. J., Suglhara, I., and Llinas, R. (1995). Dynamic organization of motor control within the olivocerebellar system. Nature 374, 453-457.

Received: 09 March 2010; paper pending published: 27 May 2010; accepted: 27 May 2010; published online: 15 September 2010.

Citation: Dias-Ferreira E, Sousa N and Costa RM (2010) Frontocerebellar connectivity: climbing through the inferior olive. Front. Neurosci. (2010) 4:37. doi: 10.3389/ fnins.2010.00037

Copyright (c) 2010 Dias-Ferreira, Sousa and Costa. This is an open-access article subject to an exclusive license agreement between the authors and the Frontiers Research Foundation, which permits unrestricted use, distribution, and reproduction in any medium, provided the original authors and source are credited. 